\title{
Monopoly-Market-Based Cooperation in Cognitive Radio Networks
}

\author{
Said Lakhal, and Zouhair Guennoun IEEE, Senior member
}

\begin{abstract}
In a cognitive radio network (CRN), the primary users (PUs) do not operate their spectra, full time. Thus, they can sell them to the secondary users (SUs), for a second use, during the free time slots. In this article, we assume that the market is perfect, monopolized by a single $\mathrm{PU}$, and all players are rational. After formulating the PU's profit, we established a necessary and sufficient condition that guarantees the introduction of the PU into the market. In addition, the expressions of the SUs' profits, showed us that in non-cooperative form, some ones got zero profit, even after maximizing their profits. Therefore, we have considered to study the effect of cooperation on the profits of this category of SUs. By following this step, we established a cooperation strategy, to avoid zero profits for all SUs. In order to analyze the impact of this cooperation on the PU, we have expressed the profits of the $P U$ in the cooperative and non-cooperative forms; as result, we found that the cooperation between SUs brought better than the non-cooperative form.
\end{abstract}

Index Terms-Cognitive radio networks, economic game, cooperation, non-cooperation, monopoly market, primary user, secondary users.

\section{INTRODUCTION}

The increase in wireless services over the last two decades has intensifies the demand for spectra. This situation forced the modification of the fixed frequency allocation policy. In 2003, the Federal Communications Commission has decided to follow a new strategy, able of managing spectra more effectively [1]. The provided efforts in this direction, have led to the emergence of the famous cognitive radio network $(\mathrm{CRN})$. This new technology found a rigid platform in the software defined radio, already designed.

Unlike the conventional networks, where the spectrum allocation is static in terms of time, space and user; the CRN offered a dynamic aspect, following all these dimensions [2]. As result, the environment dynamically changes and the users are forced to configure their settings, in order to adapt to the new changes. This type of network serves two types of customers: PUs or licensed users, who have the priority to use the spectrum, without privatization; and SUs or unlicensed users, who are waiting for the release of certain spectra, for an opportunistic use. Therefore, from time to time, some spectra are available for a second use. consequently, the spectrum sharing policy between the SUs and the comfort of the PUs

Manuscript received January 9, 2020; revised August 13, 2020. Date of publication November 3, 2020. Date of current version November 3, 2020. The associate editor prof. Adriana Lipovac has been coordinating the review of this manuscript and approved it for publication.

Authors are with the Research Team in Smart Communications-ERSC, E3S Research Center, EMI, Mohammed V University in Rabat, Morocco (e-mails: said.lakhal.rech@gmail.com,zouhair@emi.ac.ma).

Digital Object Identifier (DOI): 10.24138/jcomss.v16i4.994 represent the major challenges of CRN. Consequently, several constraints must be taken into account, when designing a CRN; among them we cite: the collision between the PUs and the SUs, energy consumption [4], interference, SUs' throughput and fairness [3], routing [5] as well as security [6]. The aims of CRN consist of overcoming these challenges, by using the available and appropriate theoretical and physical foundations. In this study, we are only interested in the theoretical bases, particularly to the application of game theory, for sharing the spectra between the unlicensed users.

The game theory is a branch of applied mathematics, developed to study the conflict and cooperation, between the rational entities, namely, players. It provides a language for formulating and analyzing the strategic scenarios. The first concepts of game theory are introduced by von Neumann and Morgenstern [7]. After, the conceptual field has been extended by the successors.

A game can be represented in the extensive or strategic form. The first is illustrated by a tree, where the nodes represent the players or the outcomes, and the branches expose the strategies or portions of probability. This type of game takes place when the players act sequentially. On the other side, the strategic form is used when the players act in parallel. It is defined by a set of players, the strategies of each player and the payoff function corresponding to both: a combination of pure strategies and a given player. When each player is assumed to know the equilibrium strategies of the other players, and no player has anything to gain, by changing only his own strategy, we talk about the famous pure strategy Nash equilibrium [8]. In the absence of this solution, we can think of a mixed strategy Nash equilibrium, by randomising over several actions of a player and looking for the solution points [9]. The games can be classified according to four families [10]: cooperative, non-cooperative, stochastic and economic games.

This work focuses on the application of an economic game on CRN, where the seller is the PU, the buyers are the SUs and the good for sale is the spectrum. This market is supposed to be perfect, because the information is accessible by all the actors. The players are considered rational, since they seek to maximize their profits. The profit of each SU is expressed by a utility function that depends on the unit price and the quantity purchased. In addition to the unit price, the PU's utility function depends on the total quantity purchased. After the maximisation of these functions, we find the quantity to buy by each SU, and the unit price specified by the PU. These results tell us that in some cases, the PU can reap zero 
profit, hence the need to establish a condition guaranteeing the participation of the PU. Likewise, some SUs can reap zero profit, when they act selfishly; but, when they cooperate, each of them will have a non-zero profit. In addition, this cooperation has a positive effect on the profit of the PU too.

The rest of this paper is organized as follows: In section II, we expose some related works treating the economic game in CRN. Section III analyses the PU's and SUs' profits. The transactions are studies in section IV. We present the simulation results in section V. We conclude in section VI. Finally, section VII is reserved to the dicussion.

\section{RELATED WORKS}

A comparison can be made between the concepts of game theory and those of CRN, as presented in table 1 .

\section{TABLE I}

CORRESPONDENCE BETWEEN GAME THEORY AND CRN [10]

\begin{tabular}{|l|l|}
\hline Game theory & CRN \\
\hline Players & Only SUs, or both SUs and PUs \\
\hline Strategies & $\begin{array}{l}\text { SUs: sensing, configuration and transmission. } \\
\text { PUs: selection of licenced bands and SUs. }\end{array}$ \\
\hline Payoff/utility function & $\begin{array}{l}\text { SUs: Increasing the QoS. } \\
\text { PUs: monitory gain. }\end{array}$ \\
\hline Cooperation or not & $\begin{array}{l}\text { SUs may cooperate to share spectra, } \\
\text { as they may selfishly behave. }\end{array}$ \\
\hline
\end{tabular}

Two wireless spectrum service providers (SSPs) competed in $[11,12]$, for attracting the end users to puchase the spectrum, in order to maximize their own profits. This situation is designed and analysed by a game model, to determine the spectrum pricing strategies and the equilibrium points. The authors of [11] treated the static game with complete information, which leaded to both market stability and pure Nash equilibrium. After, they studied a dynamic game with limited information, which provided a dynamic adjustment method and ensured the convergence to the Nash equilibrium of the sub-games. Based on the Markov chain model and queuing theory [12]; the authors demonstrated the presence and uniqueness of a sub-game perfect Nash equilibrium in the game between a SSP and its end users.

Certainly, the SUs contribute to the CRN performances, by sensing and sharing the free spectra. They can act cooperatively or selfishly. In the literature, there are many works treating these two modes, sometimes jointly and sometimes separately [13]-[16]. For the purpose of studying coverage and spectrum efficiency, as well as preventing two-level interference [13]; the authors formulated the competition case by static Cournot game with global information, and the cooperation case by Stackelberg game based on dynamic price adjustment algorithms. They proposed a new utility-driven relay scheme and introduced both: a new protocol and a refund factor. Their approach leaded at the convergence of algorithms, therefore at the existence of equilibrium. The PUs can cooperate and share the useful information, namely, the channel weights with the SUs that selfishly act in a distributed resource allocation case [14]. Based on the PUs' cooperation, the authors reduced the interference between the licensed users and unlicensed users below a threshold, whereas the selfish behavior of all SUs, leaded to a Nash Equilibrium.

Intuitively speaking, neither cooperation nor competition is extremely positive or negative: each one has its advantages and its limitations. The collaboration between SUs widened the covered region, increased the number of detected channels and ensured fairness among the unlicensed users [15]; as well as, it improved the bandwidth utilization efficiency and increased the throughput of the entire network, compared with respect to basic one-tier CRN and non-optimized twotier CRN. In [16], the authors used a game theoretical tool in the Internet of Things topic, as a result, they proved that the interactive feedback approach improved the spectrum utilization, and can be adapted to a non-cooperative repeated process. Certainly, the cooperation takes part of occupation of each user that contributes in the resolution of the collective problems. This choice could degrade the performance of some users, compared to a selfish reaction [17], where the authors applied the matching-theory-based works, by mapping the elements from two sets of equal size, based on the individual preference of the candidates. Therefore, they proved that this scheme, can be extended to match the SUs, with the channels or the PUs.

The presence of PUs, channels and SUs in the same environment, puts all in a situation similar to a market [18]. In order to guarantee a cost-effective bandwidth provisioning in Multilayer CRNs; the authors adapted news vendor model, from logistics which is compared with an adaptive period inventory management policy. After this analysis, they concluded that: it provided decision makers, with more stable supply solutions, and improved both: the total profit and user satisfaction levels.

In summary, game theory is introduced in CRN [10]-[18]. The users can cooperate as they can act selfishly. But neither cooperation nor competition is extremely positive or negative: each has its advantages and its limits [11]-[17].

Auction mechanism approach helps the SUs to get a part of the unused license band, for a lease, from the PUs [19, 20]. The authors of [19] proposed a new framwork, based on the negligible mutual interferences and satisfaction levels among the SUs, to share the leased band. Their simulation results showed that the proposed mechanism enhanced the spectral efficiency of the SUs and increased the PUs' revenues.

The vehicular networks is an environment with multi radio access, different user preferences, multiple application requirements and multiple device types. Hence, it becomes a challenge for $\mathrm{CR}$ vehicular node to select the optimal network. Highlighting, the competition between different $\mathrm{CR}$ vehicular node and access networks can be formulated as multi-bidder bidding to provide its services to $\mathrm{CR}$ vehicular node. The paper [20] proposed a new cost function based multiple attribute decision making method which outperforms other existing methods. The numerical results reveled that the proposed scheme is effective for spectrum handoff for optimal network selection among multiple available networks.

In order to make a comparison between cooperation and non-cooperation between users, the authors of [21], studied the cooperative spectrum sharing among a PU and multiple secondary users (SUs), where the PU selects a proper set 
of secondary users to serve as the cooperative relays for its transmission. They assumed that the PU and SUs are rational and selfish. As return, the PU leases portion of channel access time to the selected SUs for their transmissions, and the cooperative relays decide their respective power level used to help PU's transmission in order to achieve proportional access time to the channel. Since the SU's utility is a function of its own transmission rate and the power cost for PU's transmission, the SUs will choose a proper power level to meet the tradeoff between transmission rate and power cost. After the formulation of problem as a non-cooperative game between the PU and the SUs, the authors proved that the proposed game converges to a unique Stackelberg equilibrium.

In all these works, the PU introduced into the market, whatever the conditions, even if he will get zero profit; also, the positive impact of the cooperation between the SUs, on the PU' profit is not mentioned. Our main contributions in this work are:

1) The establishment of the necessary and sufficient condition leading to the introduction of the PU into a monopoly market.

2) The demonstration of the importance of cooperation, among the SUs who have zero profit in the non cooperative case.

3) The proof of the positive impact of cooperation between SUs on the PU's profit too.

\section{PU'S AND SU's PROFIT}

In a market, with complete information and rational players; each actor calculates his profit, before participating in a transaction. If the obtained value is greater than a threshold, the player enters into the market; otherwise, he decides not to enter. In the follows, we will calculate the profits of each SU and the PU; to determine the conditions of their participation

\section{A. SU's Profit}

Let $U_{i}$ be the profit or utility function of $S U_{i}$. It is given in [22] by:

$$
U_{i}\left(q_{i}\right)=\alpha_{i} q_{i}-\beta_{i} q_{i}^{2}-p q_{i}
$$

Such as: $\alpha_{i}, \beta_{i}, q_{i}$ and $p$ are: two real positives, purchased quantity and unit price, respectively.

$U_{i}\left(q_{i}\right)$ has a monetary unit (MU), and that of $q_{i}$ is second (s). Based on that, we can deduce the units of all defined parameters, in the next formulation part.

We assume that each $S U_{i}$ is rational: he chooses the optimal quantity $q_{i}^{*}$ that leads at the maximum profit. Since $U_{i}$ is differentiable, $q_{i}^{*}$ satisfies the optimality first condition:

$$
\begin{gathered}
U_{i}^{\prime}\left(q_{i}^{*}\right)=\alpha_{i}-2 \beta_{i} q_{i}^{*}-p=0 . \\
2 \beta_{i} q_{i}^{*}=\alpha_{i}-p, \forall i \in\{i, . ., n\} . \\
p=\alpha_{i}-2 q_{i}^{*} \beta_{i}, \quad q_{i}^{*}=\frac{\alpha_{i}-p}{2 \beta_{i}} .
\end{gathered}
$$

When we put: $\frac{1}{\beta_{i}}=\sigma_{i}$, we obtain:

$$
U_{i}\left(q_{i}^{*}\right)=\beta_{i} q_{i}^{* 2}=\frac{1}{4} \sigma_{i}\left(\alpha_{i}-p\right)^{2} .
$$

Matricially, relation (3) can be expressed by:

$$
\begin{aligned}
& \left(\alpha_{1}, ., \alpha_{n}\right)^{T}-p(1, ., 1)^{T}=\operatorname{Diag}\left(2 \beta_{1}, ., 2 \beta_{n}\right)\left(q_{1}^{*}, ., q_{n}^{*}\right)^{T} \\
& \alpha-p 1_{n}=H q^{*}
\end{aligned}
$$

With: $\alpha^{T}=\left(\alpha_{1}, ., \alpha_{n}\right), \quad 1_{n}^{T}=(1, ., 1)$,

$H=\operatorname{Diag}\left(2 \beta_{1}, ., 2 \beta_{n}\right), \quad q^{*}=\left(q_{1}^{*}, ., q_{n}^{*}\right)^{T}$.

While $\mathrm{H}$ is a diagonal matrix, with non-zero diagonal components; then, it is invertible, as result, we have:

$$
q^{*}=H^{-1} \alpha-p H^{-1} 1_{n}
$$

If we put:

$$
\begin{aligned}
\delta_{n} & =\prod_{i=1}^{n} 2 \beta_{i}=2^{n} \prod_{i=1}^{n} \beta_{i} \\
\delta_{-i} & =\prod_{\substack{j=1 \\
j \neq i}}^{n} 2 \beta_{j}=2^{n-1} \prod_{\substack{j=1 \\
j \neq i}}^{n} \beta_{j}=\frac{\sigma_{i} \delta_{n}}{2}
\end{aligned}
$$

We obtain:

$$
H^{-1}=\frac{1}{\delta_{n}} \operatorname{Diag}\left(\delta_{-1}, ., \delta_{-i}, ., \delta_{-n}\right)
$$

The second optimality condition to be checked by $q_{i}^{*}$ is: $U_{i}^{\prime \prime}\left(q_{i}^{*}\right) \leq 0$; in our case, we have:

$$
U_{i}^{\prime \prime}\left(q_{i}^{*}\right)=-2 \beta_{i}<0 .
$$

As $q_{i}^{*}$ is a critical point of $U_{i}$, i.e, $U_{i}^{\prime}\left(q_{i}^{*}\right)=0$. So, $U_{i}$ reaches its maximum at $q_{i}^{*}$.

\section{B. PU's Profit}

We assume that the unit cost is constant, it will be noted by c. Based on [22], the PU's profit will be given by:

$$
\begin{aligned}
& \Pi(p, q)=p \sum_{i=1}^{n} q_{i}-c \sum_{i=1}^{n} q_{i}=(p-c) \sum_{i=1}^{n} q_{i} \\
& \Pi\left(p, q^{*}\right)=(p-c) \sum_{i=1}^{n} q_{i}^{*}
\end{aligned}
$$

Based on relations: (6), (7) and (8); we express as follows:

$$
\begin{aligned}
\sum_{i=1}^{n} q_{i}^{*} & =(1, \ldots, 1) q^{*}=1_{n}^{T}\left(H^{-1} \alpha-p H^{-1} 1_{n}\right) \\
& =\frac{1}{\delta_{n}}\left[\sum_{i=1}^{n} \alpha_{i} \delta_{-i}-p \sum_{i=1}^{n} \delta_{-i}\right] \\
& =\frac{1}{\delta_{n}}\left[\frac{\delta_{n}}{2} \sum_{i=1}^{n} \sigma_{i} \alpha_{i}-\frac{\delta_{n}}{2} p \sum_{i=1}^{n} \sigma_{i}\right] \\
& =\frac{1}{2}\left[\sum_{i=1}^{n} \sigma_{i} \alpha_{i}-p \sum_{i=1}^{n} \sigma_{i}\right] \\
& =\frac{1}{2}\left(\gamma_{n}-p \lambda_{n}\right) . \\
\text { with: } & \gamma_{n}=\sum_{i=1}^{n} \sigma_{i} \alpha_{i}, \quad \lambda_{n}=\sum_{i=1}^{n} \sigma_{i}
\end{aligned}
$$

From (10), we obtain $\Pi$ according only to p.

$$
\Pi(p)=\frac{1}{2}(p-c)\left(\gamma_{n}-p \lambda_{n}\right)
$$


The PU exposes the price that maximizes his profit. Let $p^{*}$ be such price. Since $\Pi$ is differentiable, $p^{*}$ satisfies the first optimality condition: $\Pi^{\prime}\left(p^{*}\right)=0$.

$$
\begin{gathered}
\Pi^{\prime}\left(p^{*}\right)=\frac{1}{2}\left[\gamma_{n}+c \lambda_{n}-2 \lambda_{n} p^{*}\right]=0 \\
\Longrightarrow p^{*}=\frac{\gamma_{n}+c \lambda_{n}}{2 \lambda_{n}}=\frac{c}{2}+\frac{\gamma_{n}}{2 \lambda_{n}}
\end{gathered}
$$

The second optimality condition to be checked by $p^{*}$ is: $\Pi "\left(p^{*}\right) \leq 0$. In our case: $\Pi "\left(p^{*}\right)=-\lambda_{n}<0$.

$$
\begin{aligned}
\Pi\left(p^{*}\right) & =\frac{1}{2}\left(\frac{c}{2}+\frac{\gamma_{n}}{2 \lambda_{n}}-c\right)\left(\gamma_{n}-\left(\frac{c}{2}+\frac{\gamma_{n}}{2 \lambda_{n}}\right) \lambda_{n}\right) \\
& =\frac{1}{2}\left(\frac{\gamma_{n}}{2 \lambda_{n}}-\frac{c}{2}\right)\left(\frac{\gamma_{n}}{2}-\frac{c \lambda_{n}}{2}\right) \\
\Pi\left(p^{*}\right)=\Pi^{*}\left(\lambda_{n}, \gamma_{n}\right) & =\frac{1}{8 \lambda_{n}}\left(\gamma_{n}-c \lambda_{n}\right)^{2}
\end{aligned}
$$

From (5) and (14), the $S U_{i}{ }^{\prime} s$ profit can be written as:

$$
U_{i}\left(\lambda_{n}, \gamma_{n}\right)=\frac{\sigma_{i}}{4}\left(\alpha_{i}-p *\right)^{2}=\frac{\sigma_{i}}{4}\left[\alpha_{i}-\left(\frac{c}{2}+\frac{\gamma_{n}}{2 \lambda_{n}}\right)\right]^{2}
$$

From (15), we can easily remark that there are some combination of SUs that lead to zero PU's profit. In the following, we will propose some examples.

1) finite number of SUs

$$
\begin{aligned}
& \text { - } \beta_{1}=2, \quad \beta_{2}=3, \quad \beta_{3}=4, \quad \alpha_{1}=\frac{c}{6} \text {, } \\
& \alpha_{2}=\frac{5 c}{4}, \alpha_{3}=\frac{7 c}{3} \\
& \gamma_{3}=\sigma_{1} \alpha_{1}+\sigma_{2} \alpha_{2}+\sigma_{3} \alpha_{3}=\frac{13}{12} c, \\
& \lambda_{3}=\sigma_{1}+\sigma_{2}+\sigma_{3}=\frac{13}{12} \\
& \Pi^{*}\left(\lambda_{3}, \quad \gamma_{3}\right)=0 \\
& \text { - } \beta_{1}=2, \quad \beta_{2}=3, \quad \alpha_{1}=\frac{c}{6}, \quad \alpha_{2}=\frac{5 c}{4} \\
& \gamma_{2}=\sigma_{1} \alpha_{1}+\sigma_{2} \alpha_{2}=\frac{c}{2}, \quad \lambda_{2}=\sigma_{1}+\sigma_{2}=\frac{5}{6} \\
& \Pi^{*}\left(\lambda_{2}, \quad \gamma_{2}\right)=\frac{c^{2}}{60} \\
& \Pi^{*}\left(\lambda_{2}, \quad \gamma_{2}\right)>\Pi^{*}\left(\lambda_{3}, \quad \gamma_{3}\right)
\end{aligned}
$$

Two SUs bring more payoff than three SUs.

2) infinite number of SUs

$$
\begin{aligned}
& \alpha_{i}=c+\frac{1}{i}, \quad \beta_{i}=i, \quad i \in\{1, \ldots, n\} \\
& \lambda_{n}=\sum_{i=1}^{n} \frac{1}{i}, \quad \gamma_{n}=\sum_{i=1}^{n} \frac{\left(\frac{1}{i}+c\right)}{i} \\
& \gamma_{n}-c \lambda_{n}=\sum_{i=1}^{n} \frac{1}{i^{2}} \Longrightarrow \lim _{n \rightarrow+\infty}\left(\gamma_{n}-c \lambda_{n}\right)=\frac{\pi^{2}}{6} \\
& \lim _{n \rightarrow+\infty} \frac{1}{\lambda_{n}}=0 \Longrightarrow \lim _{n \rightarrow+\infty} \Pi^{*}\left(\lambda_{n}, \gamma_{n}\right)=0
\end{aligned}
$$

In this case, the PU have not interest to grow the number of SUs to obtain zero profit, while a fewer number returns more. From (16), we deduce that for some values of $\lambda_{n}$ and $\gamma_{n}$, we have $U_{i}\left(\lambda_{n}, \gamma_{n}\right)=0$. In this case, the $S U_{i}$ prefers to not buy spectrum.

In the next, we will study different configurations of PU's and SUs' profits, as well as, the adopted strategy by each one, for avoiding zero profit.

\section{TRansactions in a Monopoly Market}

\section{A. Removing all SUs who cancel the PU's profit}

If the PU's profit is zero, he removes one SU and after calculates his payoff with the remaining SUs. If this value equals zero, he decides to return the deleted SU, to replace him by another and calculate again the profit, and so he repeats the processsus, until a non-zero profit is met. We report that, there are some cases where such non-zero profit can not exist, whatever the deleted user. In front of this situation, instead of deleting a single $\mathrm{SU}$, the PU deletes two, based on all possible combinations, until a non-zero profit is encountered. Similarly, the obtained profits can all be equal to zero. And so, for three SUs, four..., until a combination leading to non-zero profit is encountered. The next theorem gives a necessary and sufficient condition of existence of such combination.

Theorem 1: There is a combination of SUs with which the PU's profit is non-zero if and only if there is an index $i_{0} \in\{1 \ldots n\}$, such as: $\alpha_{i_{0}} \neq c$.

\section{Proof 1:}

- We assume that there is a combination leading to nonzero PU's profit and we show that there is an index $i_{0} \in$ $\{1 \ldots n\}$, such as $\alpha_{i_{0}} \neq c$. We prove it by absurdity.

Then, we have the next logical expression:

There is a combination leading to non-zero profit and $\alpha_{i}=c, \forall i \in\{1 \ldots n\}$.

We note this combination by: $\left(S U_{k_{1}}, S U_{k_{2}}, \ldots, S U_{k_{p}}\right)$ that verifies:

$$
\Pi^{*}\left(\lambda\left(k_{1}, k_{2}, \ldots, k_{p}\right), \gamma\left(k_{1}, k_{2}, \ldots, k_{p}\right)\right) \neq 0 .
$$

We have also:

$$
\begin{aligned}
c & =\alpha_{1}=\alpha_{2}=\ldots \ldots . .=\alpha_{n} \\
& =\frac{\sigma_{1} \alpha_{1}}{\sigma_{1}}=\frac{\sigma_{2} \alpha_{2}}{\sigma_{2}}=\ldots \ldots=\frac{\sigma_{n} \alpha_{n}}{\sigma_{n}} \\
& =\frac{\sigma_{k_{1}} \alpha_{k_{1}}+\sigma_{k_{2}} \alpha_{k_{2}}+\ldots+\sigma_{k_{p}} \alpha_{k_{p}}}{\alpha_{k_{1}}+\alpha_{k_{2}}+\ldots+\alpha_{k_{p}}} \\
& =\frac{\gamma\left(k_{1}, k_{2}, \ldots, k_{p}\right)}{\lambda\left(k_{1}, k_{2}, \ldots, k_{p}\right)}
\end{aligned}
$$

By referring to relation (15), we deduce: $\Pi^{*}\left(\lambda\left(k_{1}, k_{2}, \ldots, k_{p}\right), \gamma\left(k_{1}, k_{2}, \ldots, k_{p}\right)\right)=0$.

It is a contradiction with (17).

- We assume that there is an index $i_{0} \in\{1 \ldots n\}$, such as $\alpha_{i_{0}} \neq c$ and we show that there is a combination leading to non-zero PU's profit.

Trivial, just take the combination that contains only $S U_{i_{0}}$, and the result will be immediate.

Therefore, it is sufficient to have a single $S U_{i_{0}}$, such that $\alpha_{i_{0}} \neq$ $c$, for the PU to enter into the market.

To make his decision, the PU calculates the decision index:

$$
d=\sum_{i=1}^{n}\left|\alpha_{i}-c\right|
$$

If $d$ is different to zero, he enters, otherwise he will not enter. In the rest of this paper, we consider that $d \neq 0$. Therefore, after deleting all SUs who cancel his profit, the PU will 
keep a combination with which will have non-zero profit, i.e. $\Pi^{*}\left(\lambda_{n}, \gamma_{n}\right) \neq 0 \Longleftrightarrow \frac{\gamma_{n}}{\lambda_{n}} \neq c$.

In the following, we consider that:

$$
\frac{\gamma_{n}}{\lambda_{n}} \neq c
$$

\section{B. SU's strategy}

From (16), we have:

$$
U_{i}^{*}\left(q_{i}^{*}\right)=0 \Longleftrightarrow \alpha_{i}=\frac{c}{2}+\frac{\gamma_{n}}{2 \lambda_{n}}
$$

The SU does not have interest to play for harvesting zero profit. Thus, all SUs who have zero profit retire one after one, to leave in the market only those who have non-zero profit.

\section{Interaction between SUs}

We note that this interaction takes place in the presence of at least two SUs having together zero profit.

We are in front of three cases:

- Case1: In the market, There are exactly two SUs, each one has zero parofit:

Let $S U_{1}\left(\alpha_{1}, \beta_{1}\right), S U_{2}\left(\alpha_{2}, \beta_{2}\right)$ be such users. Based on relation (16), we can write:

$$
\alpha_{1}=\alpha_{2}=\frac{c}{2}+\frac{\gamma_{2}}{2 \lambda_{2}} \Longleftrightarrow \alpha_{1}=\alpha_{2}=c
$$

Each user has two possible strategies: participate $(\mathrm{P})$ in the game, i.e. buying a spectrum, or not participate (NP). In this case, regardless of the strategy of each player, they always have zero payoff.

- Case2: They are more than two SUs in the market, among them, two have zero payoff.

Based on relation (16), we can write:

$$
\alpha_{1}=\alpha_{2}=\frac{c}{2}+\frac{\gamma_{n}}{2 \lambda_{n}}
$$

We will calculat the payoff of $S U_{2}$, after retrait of $S U_{1}$

$$
\begin{aligned}
U_{2}^{*}\left(q_{2}^{*}\right) & =\frac{\sigma_{2}}{4}\left[\alpha_{2}-\left(\frac{c}{2}+\frac{\gamma_{n}-\sigma_{1} \alpha_{1}}{2\left(\lambda_{n}-\sigma_{1}\right)}\right)\right]^{2} \\
& =\frac{\sigma_{2}}{4}\left[\frac{c}{2}+\frac{\gamma_{n}}{2 \lambda_{n}}-\left(\frac{c}{2}+\frac{\gamma_{n}-\sigma_{1} \alpha_{1}}{2\left(\lambda_{n}-\sigma_{1}\right)}\right)\right]^{2} \\
& =\frac{\sigma_{2}}{4}\left[\frac{\gamma_{n}}{2 \lambda_{n}}-\frac{\gamma_{n}-\sigma_{1} \alpha_{1}}{2\left(\lambda_{n}-\sigma_{1}\right)}\right]^{2} \\
& =\frac{\sigma_{2}}{4}\left[\frac{\sigma_{1}}{2\left(\lambda_{n}-\sigma_{1}\right)}\left(\alpha_{1}-\frac{\gamma_{n}}{\lambda_{n}}\right)\right]^{2} \\
& =\frac{\sigma_{2} \sigma_{1}^{2}}{16\left(\lambda_{n}-\sigma_{1}\right)^{2}}\left[c-\frac{\gamma_{n}}{\lambda_{n}}\right]^{2} \\
& =\theta_{2}>0 \quad(\text { condition }(19))
\end{aligned}
$$

Similary, we obtain:

$$
U_{1}^{*}\left(q_{1}^{*}\right)=\frac{\sigma_{1} \sigma_{2}^{2}}{16\left(\lambda_{n}-\sigma_{2}\right)^{2}}\left[c-\frac{\gamma_{n}}{\lambda_{n}}\right]^{2}=\theta_{1}>0 .
$$

We note by $P_{1}\left(s_{1}, s_{2}\right)$ and $P_{2}\left(s_{1}, s_{2}\right)$, the $S U_{1}$ 's and $S U_{2}$ 's profit, when $S U_{1}$ and $S U_{2}$ adopt strategies $s_{1}$ and $s_{2}$, respectively. Knowing that: $\left(s_{1}, s_{2}\right) \in\{P, N P\}^{2}$.
Based on (23) and (24), table II presents the $S U_{1}^{\prime} s$ and $S U_{2}^{\prime} s$ profit in the non-cooperative case, according to the adopted strategy by each one.

TABLE II

$S U_{1}^{\prime} s$ AND $S U_{2}^{\prime} s$ PROFIT, IN THE NON-COOPERATIVE MODE

\begin{tabular}{|l|c|c|}
\hline$S U_{1} S U_{2}$ & $\mathrm{P}$ & $\mathrm{NP}$ \\
\hline $\mathrm{P}$ & $(0,0)$ & $\left(\theta_{1}, 0\right)$ \\
\hline $\mathrm{NP}$ & $\left(0, \theta_{2}\right)$ & $(0,0)$ \\
\hline
\end{tabular}

The SUs can cooperate to avoid zero payoff for both $S U_{1}$ and $S U_{2}$. In this situation, there are only two possible couples of strategies: (P, NP) and (NP, P).

Then, let $P_{1}^{c}$ and $P_{2}^{c}$ be the $S U_{1}^{\prime} s$ and $S U_{2}^{\prime} s$ payoff, respectively; such as:

$P_{1}^{c}(P, N P)=\frac{\theta_{1}^{2}}{\theta_{1}+\theta_{2}}, \quad P_{2}^{c}(P, N P)=\frac{\theta_{1} \theta_{2}}{\theta_{1}+\theta_{2}}$,

$P_{1}^{c}(N P, P)=\frac{\theta_{1} \theta_{2}}{\theta_{1}+\theta_{2}}, \quad P_{2}^{c}(P, N P)=\frac{\theta_{2}^{2}}{\theta_{1}+\theta_{2}}$.

Table III exposes the profit of each SU.

TABLE III

$S U_{1}^{\prime} s$ AND $S U_{2}^{\prime} s$ PAYOFF, IN THE COOPERATIVE MODE

\begin{tabular}{|l|c|c|}
\hline$S U_{1} S U_{2}$ & $\mathrm{P}$ & $\mathrm{NP}$ \\
\hline $\mathrm{P}$ & IIIIIIIIIIIIIIIIIIIIIII & $\left(\frac{\theta_{1}^{2}}{\theta_{1}+\theta_{2}}, \frac{\theta_{1} \theta_{2}}{\theta_{1}+\theta_{2}}\right)$ \\
\hline $\mathrm{NP}$ & $\left(\frac{\theta_{1} \theta_{2}}{\theta_{1}+\theta_{2}}, \frac{\theta_{2}^{2}}{\theta_{1}+\theta_{2}}\right)$ & |IIIIIIIIIIIIIIIIIIIIII \\
\hline
\end{tabular}

If we put: $\theta=\max \left(\theta_{1}, \theta_{2}\right)$, we have:

$\frac{\theta \theta_{1}}{\theta_{1}+\theta_{2}}=\max \left(\frac{\theta_{1}^{2}}{\theta_{1}+\theta_{2}}, \frac{\theta_{1} \theta_{2}}{\theta_{1}+\theta_{2}}\right)=\max _{s_{1}, s_{2}} P_{1}^{c}\left(s_{1}^{c}, s_{2}^{c}\right)$,

$\frac{\theta \theta_{2}}{\theta_{1}+\theta_{2}}=\max \left(\frac{\theta_{2}^{2}}{\theta_{1}+\theta_{2}}, \frac{\theta_{1} \theta_{2}}{\theta_{1}+\theta_{2}}\right)=\max _{s_{1}, s_{2}} P_{2}^{c}\left(s_{1}^{c}, s_{2}^{c}\right)$.

As result, both players have an interest in removing the player who has a minimum payoff in the non-cooperative game, and in participating who has the maximum payoff.

- Case3: Currently, we generalize for k SUs. We denote by: $S U_{1}, \ldots, S U_{k}$ these users.

By applying relation (16), we can write:

$$
\alpha=\alpha_{1}=\alpha_{2}=\ldots . .=\alpha_{k}=\frac{c}{2}+\frac{\gamma_{n}}{2 \lambda_{n}}
$$

We will calculate:

$$
\begin{aligned}
& U_{1}^{*}\left(q_{1}^{*}\right) \text {, if } \forall j \in\{2, \ldots, k\}, \quad S U_{j} \quad \text { is removed. } \\
& U_{1}^{*}\left(q_{1}^{*}\right)=\frac{\sigma_{1}\left(\sum_{j=2}^{k} \sigma_{j}\right)^{2}}{16\left(\lambda_{n}-\sum_{j=2}^{k} \sigma_{j}\right)^{2}}\left(c-\frac{\gamma_{n}}{\lambda_{n}}\right)^{2}=\theta_{1}>0 .
\end{aligned}
$$

Sumilary, if:

$\forall j \in\{1 \ldots k\}$ and $j \neq l, \quad S U_{j}$ is removed, we find:

$$
U_{l}^{*}\left(q_{l}^{*}\right)=\frac{\sigma_{l}\left(\sum_{\substack{j=1 \\ j \neq l}}^{k} \sigma_{j}\right)^{2}}{16\left(\lambda_{n}-\sum_{\substack{j=1 \\ j \neq l}}^{k} \sigma_{j}\right)^{2}}\left(c-\frac{\gamma_{n}}{\lambda_{n}}\right)^{2}=\theta_{l}>0 .
$$

Therefore, we have:

$P_{l}\left(s_{1}, \ldots, s_{k}\right)>0 \Leftrightarrow s_{l}=P, s_{j}=N P, \quad \forall j \neq l$.

Then, in the non-cooperative game, only the remaining player, after the deletions of all players who have zero payoff, will participe in the market. 
Currently, we examine the cooperative case, when the players negotiate, in order to have non-zero win and to share the winnings according to the contribution of each one.

The $S U_{j}{ }^{\prime} s$ payoff is given by:

$$
P_{j}^{c}=\frac{\theta \theta_{j}}{\sum_{l=1}^{k} \theta_{l}}, \quad \text { with: } \quad \theta=\max \left\{\theta_{l} / l \in\{1, \ldots, k\}\right\} .
$$

Knowing that, the user who wins the maximum gain in the non-cooperative game will play, and the others leave the game.

From relation (26), we can easily extract three remarks: 1) $P_{j}^{c}<\theta_{j}$ : the $S U_{j}{ }^{\prime} s$ payoff in non-cooperative game is greater than that in the cooperative game.

2) $\sum_{j=1}^{k} P_{j}^{c}=\theta$ : the share of win between all SUs.

3) $P_{j}^{c} \geq \frac{\theta_{i} \theta_{j}}{\sum_{l=1}^{k} \theta_{l}}: S=(N P, \ldots, P, N P . ., N P)$,

with: $S_{l} \stackrel{l=1}{=} P$ and $\theta_{l}=\theta$, is the dominant strategy for the cooperative game.

To compare non-cooperative and cooperative games, we calculate the average number $A$ of SUs that have non-zero gain.

$$
A=\frac{\text { Total nomber of users having non-zero payoff }}{\text { Total number of SUs }}
$$

For $k$ users, in the non-cooperative and cooperative games, we have: $A^{n c}=\frac{k}{k+1}$ and $A^{c}=k$, respectively.

We can easily remark that $A^{c}>>A^{n c}$.

\section{Impact of cooperation on the PU's payoff}

From equation (15) we have: $\Pi^{*}\left(\lambda_{n}, \gamma_{n}\right)=\frac{1}{8 \lambda_{n}}\left(\gamma_{n}-c \lambda_{n}\right)^{2}$. Let $\Pi_{N C}^{*}\left(\lambda_{n}, \gamma_{n}\right)$ and $\Pi_{C}^{*}\left(\lambda_{n}, \gamma_{n}\right)$ be, the PU's payoff in the non-cooperative and cooperative cases, respectively. Then:

$$
\begin{aligned}
\Pi_{N C}^{*}\left(\lambda_{n}, \gamma_{n}\right) & =\frac{1}{8 \lambda_{n}}\left(\gamma_{n}-c \lambda_{n}\right)^{2}=\frac{\lambda_{n}}{2}\left(\alpha_{i}-c\right)^{2} \\
& =\frac{\lambda_{n}}{2}(\alpha-c)^{2} .
\end{aligned}
$$

To simplify the calculation, we use relation (25) and we put: $\Sigma=\sum_{i=1}^{k} \sigma_{i}$

$$
\begin{aligned}
\Pi_{C}^{*}\left(\lambda_{n}, \gamma_{n}\right) & =\frac{1}{8\left(\lambda_{n}-\Sigma\right)}\left[\gamma_{n}-\sum_{i=1}^{k} \sigma_{i} \alpha_{i}-c\left(\lambda_{n}-\Sigma\right)\right]^{2} \\
& =\frac{1}{8\left(\lambda_{n}-\Sigma\right)}\left[\gamma_{n}-c \lambda_{n}+(c-\alpha) \Sigma\right]^{2} \\
& =\frac{1}{8\left(\lambda_{n}-\Sigma\right)}\left[\gamma_{n}-c \lambda_{n}+(c-\alpha) \Sigma\right]^{2} \\
& =\frac{(\alpha-c)^{2}}{8\left(\lambda_{n}-\Sigma\right)}\left(2 \lambda_{n}-\Sigma\right)^{2}
\end{aligned}
$$

Let $\mathrm{f}$ be the function defined by:

$$
\begin{aligned}
f: \quad] \Sigma,+\infty[ & \longrightarrow \mathbb{R}_{+}, \\
x & \longrightarrow \frac{(\alpha-c)^{2}}{8(x-\Sigma)}(2 x-\Sigma)^{2}
\end{aligned}
$$

Properties of function $\mathrm{f}$ :

$\mathrm{P} 1: \mathrm{f}$ is decreasing on $\left.] \Sigma, \frac{3 \Sigma}{2}\right]$ and increasing on $\left[\frac{3 \Sigma}{2},+\infty[\right.$.

P2: At the neighborhood of $+\infty, \quad f(x) \approx \frac{(\alpha-c)^{2}}{2} x$.

P3: $\left.f(x) \geq \frac{(\alpha-c)^{2}}{2} x, \quad \forall x \in\right] \Sigma,+\infty[$.

Then, from (27), (28) and P3 we have:

$$
\Pi_{C}^{*}\left(\lambda_{n}, \gamma_{n}\right) \geq \Pi_{N C}^{*}\left(\lambda_{n}, \gamma_{n}\right) .
$$

As result, the cooperation between SUs is in the interest of the PU too.

\section{Simulations}

\section{A. SUs' performances}

In the purpose to compare the SUs' profits in the cooperative case with those in the non-cooperative case, we consider some number of SUs, and we calculate the values of $A$. Fig. 1 illustrates this comparison.

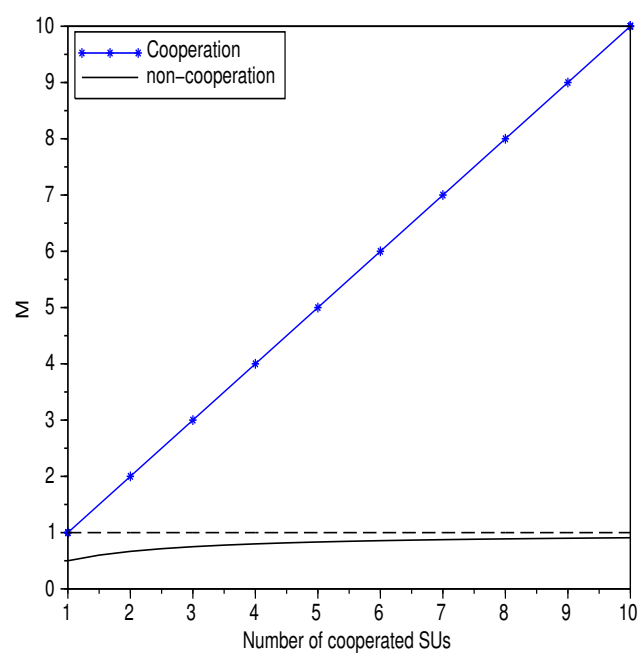

Fig. 1. Comparison between cooperative and non-cooperative games

We can easily remark that the values of $A$ in the cooperative case are very important comparing to those in the noncooperative case. This pheromone can be explained by the massif increase of the number of SUs who have a nonzero profit in the cooperative case. Moreover, this cooperation allows SUs that have zero profit in the non-cooperative form to earn non-zero profit.

\section{B. PU's performance}

In the objective to compare the PU's profit in the cooperative case with that in the non-cooperative case, we take some value of $\lambda$ and we calculate the PU's profit. Fig. 2 exposes the obtained results.

We conclude that the curve of the cooperative case is always on that of the non-cooperative case, and at the infinity, the two curves approach. The participation of SUs that have zero profit, decreases PU's profit; because their role is destructive in the market. Therefore, their withdrawal gives more profit to other players. 


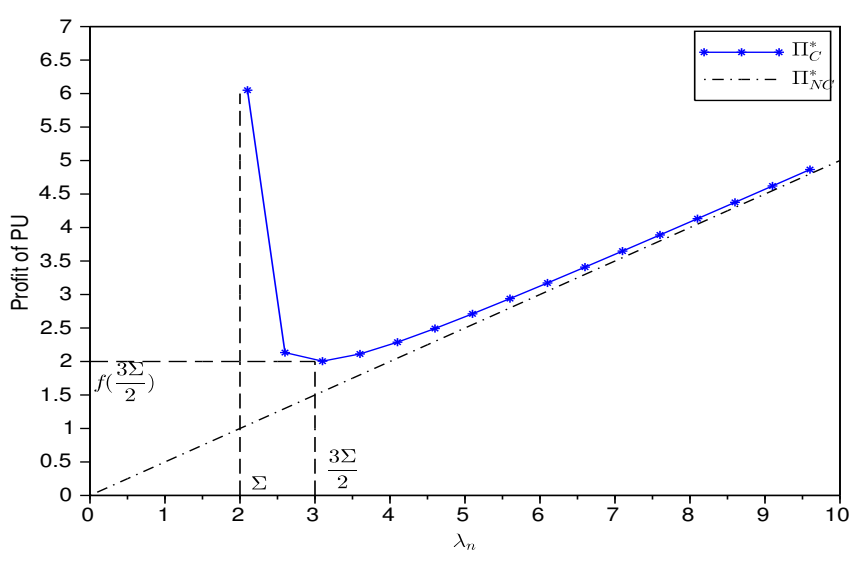

Fig. 2. PU's profit in the cooperative and non-cooperative modes

\section{Comparison between our performances and those of [19]}

In order to compare our performances in the cooperative form with those of [19] based on table 1.1 and table 1.2 of the mentioned paper, we take the same number of SUs and the same cost $c=30$. After the evaluation of the spectral efficiency, and the PU's profit, we obtain tables IV and V, respectively.

TABLE IV

COMPARISON BETWEEN OUR MEDEL AND THAT OF [19] IN TERMS OF SPECTRUM EFFICIENCY

\begin{tabular}{|l|l|l|}
\hline Number of SUs & Model of [19] & Our model \\
\hline 8 & 75 & 65 \\
\hline 10 & 95 & 80 \\
\hline 12 & 110 & 100 \\
\hline 14 & 120 & 130 \\
\hline 16 & 128 & 143 \\
\hline 18 & 130 & 152 \\
\hline 20 & 170 & 181 \\
\hline
\end{tabular}

TABLE V

COMPARISON BETWEEN OUR MEDEL AND THAT OF [19] IN TERMS OF PU'S PROFIT

\begin{tabular}{|l|l|l|}
\hline Number of SUs & Model of [19] & Our model \\
\hline 8 & 72.5 & 68 \\
\hline 10 & 94.5 & 88 \\
\hline 12 & 110 & 115 \\
\hline 14 & 116. & 121 \\
\hline 16 & 123.5 & 128 \\
\hline 18 & 131.5 & 133 \\
\hline 20 & 165 & 175 \\
\hline
\end{tabular}

The results of such tables, can be represented graphically by fig. 3 and fig. 4 .

We remark that for the smallest number of SUs, the performance of [19] is better than ours; but for the higher number, our results exceed those of [19]. This phenomenon can be explained by the increase in overall profit, when the number of cooperated SUs increases, and will be the same for the PU.

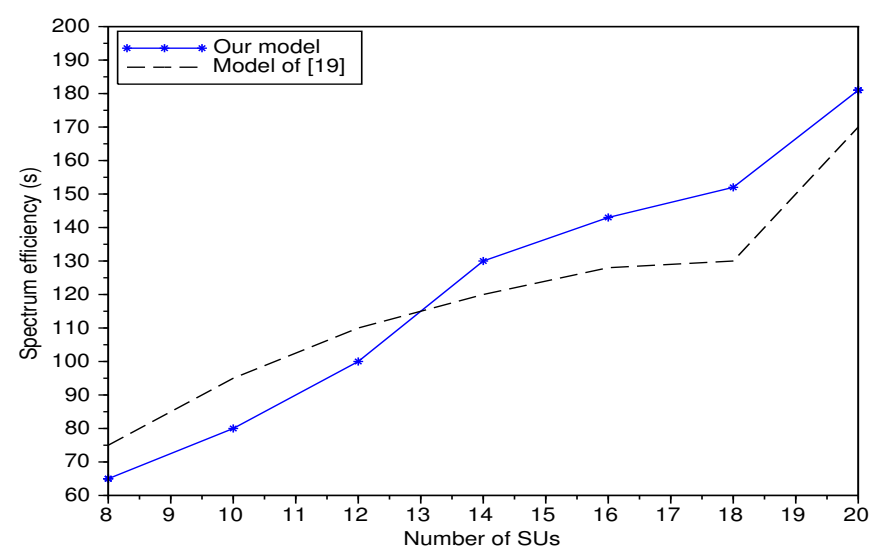

Fig. 3. Comparison between our model and that of [19] in terms of spectrum efficiency

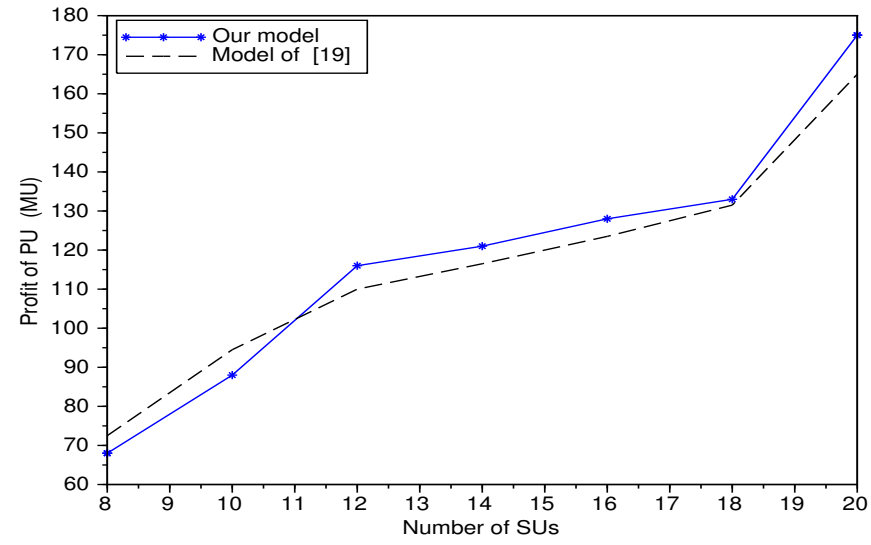

Fig. 4. Comparison between our model and that of [19] in terms of PU's profit

\section{CONCLUSION}

In this work, we studied the process of selling spectra by a PU to the SUs. This market is supposed to be monopolized by a single vendor, and the information is considered complete and available to all actors who act rationally.

We have shown that even after maximizing the profits of the PU or SUs, the obtained values can remain zero, and thus the player has not benefited by his introduction into the market. The study of the PU's profit allows us to the establishment of a necessary and sufficient condition on the actors' parameters for the held of the market. On the other hand, the analysis of the SUs' profits showed us that those who have zero profit can avoid this situation by cooperating. Also, this cooperation is in the interest of the PU too. All the work is based on perfect information, where the players share the useful information. In the case of non-perfect information, two scenarios can be considered: Cooperative scenario: the players who share the same information will be grouped together in the same cluster, to cooperate to estimate the missing information. Through this policy, the members of the same group reduce the level of risk. 
In non-cooperation scenario, the market can be organized as an auction game; thus, the player with the best price buys the spectrum.

\section{DISCUSSION}

Cooperation between SUs adds costs to pay, especially in terms of three parameters: the benefits of some SUs, algorithmic complexity and energy consumption. First, the SU who has the maximum profit and participates in the cooperation, he shares his profit with other SUs, and thus his initial profit is reduced. Second, cooperation requires the identification of all SUs, those with zero profit and the one with maximum profit. After the participation of that particular SU, the profit calculation of each SU is done, to share the overall profit. Trivially, all these operations increase the algorithmic complexity compared to the non-cooperative form. Third, cooperation between actors requires the exchange, memorization and processing of data, via communication, storage and treatments devices. These equipments require power to operate; consequently, the power consumption will be increased. As result, even the positive impact of cooperation, this strategy has its costs in terms of some SUs' benefits, computing time, and power consumption.

\section{REFERENCES}

[1] I.Mustapha, B.M.Ali, A.Sali, M.F.A.Rasid, H.Mohamad, "An energy efficient Reinforcement Learning based Cooperative Channel Sensing for Cognitive Radio, Sensor Networks", J. Pervasive and Mobile Computing, 2016. doi: 10.1016/j.pmcj.2016.07.007.

[2] J.Mitola III, Cognitive Radio An Integrated Agent Architecture for Software Defined Radio, $\mathrm{PhD}$ dissertation, Royal Institute of Technology (KTH), Kista Sweden, 2000. doi:10.4236/cn.2013.53B205.

[3] X.Liu and M.Jia, "Joint optimal fair cooperative spectrum sensing and transmission in cognitive radio", J. Physical Communication, 2017. doi: 10.1016/j.phycom.2017.08.015.

[4] J.Guo, H.Hu, X.Da, J.Liu, W.Li, "Optimization of Energy Efficiency for Cognitive Radio with Partial RF Energy Harvesting", J. International Journal of Electronics and Communications, 2017. doi: 10.1016/j.aeue.2017.12.030.

[5] J.S.P.Singh, M.K.Rai, "Cognitive Radio Routing Protocol for Link Quality Channel Diverse Cognitive Networks", J. Journal of Network and Computer Applications, 2017. doi: 10.1016/j.jnca.2017.12.014.

[6] M.Bouabdellah, N.Kaabouch, F.E.Bouanani, H.B.Azzac, "Network layer attacks and countermeasures in cognitive radio networks: A survey", J. Journal of Information Security and Applications, 2017. doi: 10.1016/j.jisa.2017.11.010.

[7] J.v.neuman and O.Morgenstern, Theory of games and economic behavior, Princeton University Press, 1945. doi: 10.2307/1232672.

[8] M.J. Osborne and A.Rubinstein, A Course in Game Theory, Cambridge Massachusetts Institute of Technology, 2011. doi:10.2307/136062.

[9] C.Y.Lee, "Mixed-Strategy Nash Equilibrium in Data Envelopment Analysis", J. European Journal of Operational Research, 2017. doi: 10.1016/j.ejor.2017.10.048.

[10] B.Wang, et al, "Game theory for cognitive radio networks: An overview", J. Computer Networks 2010. doi: 10.1016/j.comnet.2010.04.004.

[11] J.Jia and Q.Zhang, "Competitions and Dynamics of Duopoly Wireless Service Providers in Dynamic Spectrum Market", in 2008 Int. Conf. MobiHoc08, 2008. doi: 10.1145/1374618.1374660.

[12] L.Wang, et al, "Game Theory-Based Model for Maximizing SSP Utility in Cognitive Radio Networks", J. Computer Communications, 2016. doi: 10.1016/j.comcom.2016.04.002.

[13] L.G.Yi and Y.M.Lu, "Utility-driven relay for hybrid access femtocells based on cognitive radio spectrum auction", J. Computer Networks, 2015 doi: 10.1016/j.comnet.2015.01.014.

[14] P.D.Mankar, et al, "Semi-cooperative game theoretic framework for resource allocation in cognitive cellular networks", J. Physical Communication, 2018. doi: 10.1016/j.phycom.2018.01.005.
[15] A.Roy, et al, "Optimized Secondary User Selection for Quality of Service Enhancement of Two-tier Multi-User Cognitive Radio Network: A Game Theoretic Approach", J. Computer Networks, 2017. doi: 10.1016/j.comnet.2017.05.002.

[16] S.Kim, "Inspection game based cooperative spectrum sensing and sharing scheme for cognitive radio IoT system", J. Computer Communications, 2017. doi: 10.1016/j.comcom.2017.01.015.

[17] S.Chowdhury, Matching theory for cognitive radio networks: An overview, The Korean Institute of Communications Information Sciences, 2018. doi: 10.1016/j.icte.2018.01.008.

[18] G.Aloi, "On the economic sustainability of supplying bandwidth policies in multi-layer wireless cognitive networks", J. Applied Mathematical Modelling, 2016. doi: 10.1016/j.apm.2015.12.036.

[19] D.S.Sofia and A.S.Edward, "Auction based game theory in cognitive radio networks for dynamic spectrum allocation", J. Computers and Electrical Engineering, vol. 86, 2020. doi: 10.1016/j.compeleceng.2020.106734.

[20] K.Kumar, A.Prakash and R.Tripathi,"A Spectrum Handoff Scheme For Optimal Network Selection in Cognitive Radio Vehicular Networks: A Game Theoritic Auction Theory Approach", J.Physical Communication, 2017. doi: 10.1016/j.phycom.2017.04.001.

[21] H.Wang, L.Gao, X.Gan, X.Wang, and E.Hossain,"Cooperative Spectrum Sharing in Cognitive Radio Networks: A Game-Theoretic Approach", In IEEE ICC proceedings, 2010. doi: 10.1109/ICC.2010.5502052.

[22] N.Singh and X.Vives, "Price and quantity competition in a differentiated duopoly", Rand Journal of Economics Vol. 15, No. 4, 1984. doi: $10.1111 /$ jere. 12109 .

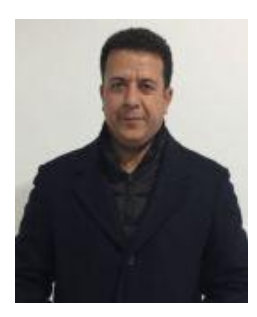

Said Lakhal obtained the diploma of application engineer in computer sciences in 1998, from the University Sidi Mohamed Ben Abdelah, Fes, Morocco. M.Sc. degree in modelization in 2006, from Mohammadia School of Engineering. He is currently a member of Team in Smart CommunicationsERSC, E3S Research Center, EMI, Mohammed V University in Rabat, Morocco. His current research interests are Computing, Cognitive Radio, WBAN, IoT, Algorithmic and Complexity.

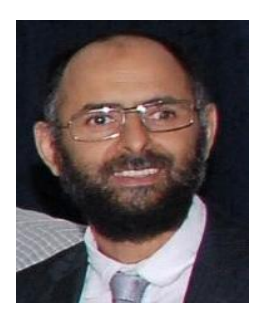

Zouhair Guennoun was born in Fès, Morocco in 1964. He received his engineering degree in Electronics and Telecommunications from the Electronics and Electrical Montefiore Institute, ULG Liege, Belgium in 1987; his M.Sc. degree in Communication Systems from the EMI School of Engineering, Rabat, Morocco in 1993; and his $\mathrm{PhD}$ degree from the same school in 1996. He visited the Centre for Communication Research (CCR) in Bristol University, UK, during the period of 1990-1994 to prepare a split $\mathrm{PhD}$. His fields of interest are digital signal processing, error control coding, speech and image processing, telecommunication systems, networks architecture and networks security. Prof. Guennoun is an IEEE senior member (member since 1990); and an ex-member of the Moroccan IEEE section executive committee. During 1988-1996 he worked as an Assistant Lecturer in the EMI School of engineering, and from 1996 he is working in the same school as a Professor Lecturer. Currently, he is in charge of the research team of Smart Communications-ERSC (formerly known as LEC) as part of the research center in engineering sustainable and smart systems at EMI, University Mohammed V in Rabat. 